\title{
Effect of Ambient Pressure on Micro-Explosion of an Emulsion Droplet Evaporating on a Hot Surface*
}

\author{
Hajime TANAKA ${ }^{* *}$, Toshikazu KADOTA**, Daisuke SEGAWA**, \\ Shinji NAKAYA** and Hiroshi YAMASAKI***
}

\begin{abstract}
An experimental study has been carried out to reveal the statistical characteristics for the onset of micro-explosion of an emulsion droplet evaporating on a hot surface. The measurements are made of the waiting time for the onset of micro-explosion at various ambient pressures, base fuels, water contents and surface temperatures. The Weibull analysis is applied to obtain the distribution function of the waiting time for the onset of micro-explosion and to derive the empirical formula for the rate of micro-explosion as a function of the water volume and emulsion temperature. The results show that the waiting time is correlated well with the Weibull distribution of the wear-out type. The waiting time decreases with an increase in the ambient pressure, the saturation temperature of base fuel, the water content and the surface temperature. An empirical formula is proposed for the rate of micro-explosion as a function of the water volume and emulsion temperature.
\end{abstract}

Key Words: Liquid Fuel, Evaporation, Diesel Engine, Emulsion Droplet, Micro-Explosion, Hot Surface, High Pressure, Weibull Distribution

\section{Introduction}

The evaporation of emulsion which consists of base fuel and water doped with a small amount of surfactant is frequently accompanied by the micro-explosion which is caused by the vigorous phase change of interior water and subsequently by the secondary atomization. It is well known that the secondary atomization improves the mixing of fuel and air and helps to suppress the formation of nitric oxides, soot and unburned hydrocarbons. In the combustion chambers of diesel engines and gas turbines, a part of fuel spray supplied in high pressure and high temperature gaseous environments is allowed to impact on a hot surface and evaporates. The evaporation phenomena of the emulsion droplet on a hot surface plays an important role in the process of fuel-air mixing, combustion and formation of undesirable pollutants in these engines.

Although a lot of works have been done on the evap-

* Received 13th July, 2006 (No. 05-0559). Japanese Original: Trans. Jpn. Soc. Mech. Eng., Vol.72, No.713, B (2006), pp.138-143 (Received 19th May, 2005)

** Department of Mechanical Engineering, Osaka Prefecture University, 1-1 Gakuen-cho, Naka-ku, Sakai-shi, Osaka 599-8531, Japan. E-mail: kadota@me.osakafu-u.ac.jp

*** College of Industrial Technology, Nihon University, 1-21 Izumi-cho, Narashino-shi, Chiba 275-0006, Japan oration and combustion of emulsion droplet ${ }^{(1)-(3)}$, a limited number of papers has been reported on the emulsion droplet evaporating and burning on a hot surface including the experimental works by Kimoto et al. ${ }^{(4)}$, Yoshimoto et al. ${ }^{(5)}$, Averdisian et al. ${ }^{(6)}$ and Chung et al. ${ }^{(7)}$ Primary attention in these works has been toward the phenomenological description of the evaporation behavior of a droplet on a hot surface. There is a surprising lack of understanding of the fundamental aspect of the processes occurring inside the emulsion droplet. The complicated processes associated with the onset of micro-explosion of emulsion droplet which is ruled by the heat and mass transfer inside the liquid phase of water and base fuel, the nucleation of micro-vapor bubbles and its growth remain largely unexplored.

The authors have been involved in a series of fundamental studies to make a deep insight into the onset of micro-explosion of an emulsion droplet evaporating and burning on a hot surface. As a first step, the effect of the water content, the properties of base fuels and the surface temperature on the statistical characteristics of the onset of micro-explosion has been studied in the previous works $^{(8),(9)}$.

The primary objective of the present study is to elucidate the effect of the ambient pressure on the onset of micro-explosion of a droplet of oil-in-water $(\mathrm{O} / \mathrm{W})$ type 
emulsion evaporating on a hot surface. Photographic observations and measurements of the waiting time for the onset of micro-explosion are made for a droplet of an initial diameter of $2 \mathrm{~mm}$ at the ambient pressures ranging from $0.1 \mathrm{MPa}$ to $0.3 \mathrm{MPa}$. The primary attention is paid on the statistical analysis to derive the distribution function of the waiting time for the onset of micro-explosion which is affected by the ambient pressure, the base fuel properties, the water content and the surface temperature.

\section{Experimental Apparatus and Procedure}

Figure 1 shows the schematic diagram of the experimental apparatus. The apparatus consists of a high pressure chamber in which an emulsion droplet is allowed to evaporate, a fuel supply system, an emulsion droplet, a stainless steel block, an electric heater, thermocouples, a video camera, a recording system and a high pressure nitrogen cylinder. The cylindrical high pressure chamber made of duralumin has the dimensions of $100 \mathrm{~mm}$ inner diameter and $250 \mathrm{~mm}$ inner height. In order to observe the emulsion droplet, a pair of circular glass windows is equipped on the sidewalls of the chamber. The upper flange of the chamber is equipped with the fuel supply system and a cooling system. The cylindrical stainless steel block having a diameter of $40 \mathrm{~mm}$ and a height of $50 \mathrm{~mm}$ occupies the lower portion of the chamber. The test surface of the stainless steel block is machined to be a part of a sphere of a diameter of $100 \mathrm{~mm}$ and polished to a mirror finish. It is surrounded by an annular electric heater. The annular space between the heating block and the inner wall of the high pressure chamber is filled with a heat insulator. A cooling water line is equipped to suppress the temperature rise of the chamber wall. The temperature of the test surface is determined by extrapolating the temperatures measured by using three thermocouples of a diameter of $0.1 \mathrm{~mm}$, whose hot junctions are embedded within the heating block at vertical spacings of $5 \mathrm{~mm}$ along its central axis.

A droplet of oil-in-water $(\mathrm{O} / \mathrm{W})$ type emulsion is

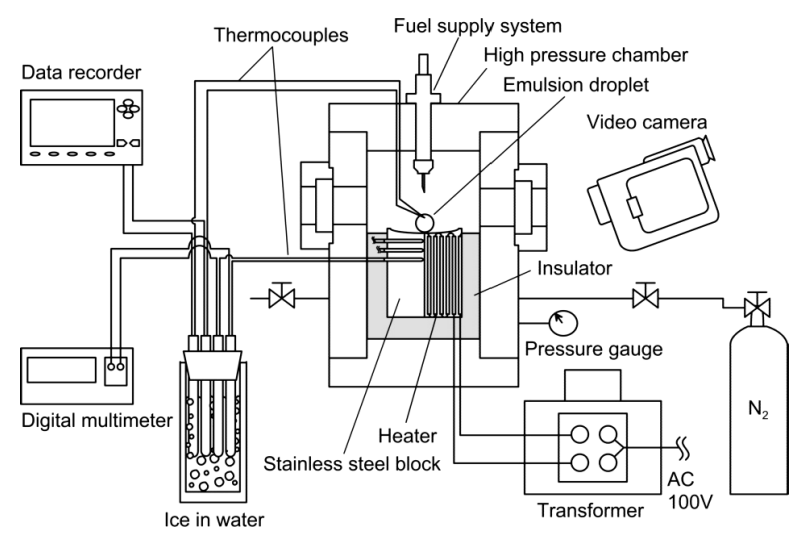

Fig. 1 Experimental apparatus tested, which consists of base fuel, water and a small amount of surfactant. The base fuels employed are $n$ dodecane (normal boiling point: $489.5 \mathrm{~K}$ ), $n$-tetradecane $(526.7 \mathrm{~K})$ and $n$-hexadecane $(560 \mathrm{~K})$. Polyoxyethylene nonylphenyl ether (Emulgen 909, Kao Corp., HLB = 12.4) is added as a surfactant whose volumetric concentration is kept at $1 \%$. Base fuel, water and surfactant are mixed well with the aide of a magnetic stirrer to make an emulsion. The emulsion is degassed by keeping it under reduced the ambient pressure at about $5 \mathrm{kPa}$ for 1 hour prior to being tested.

The chamber is pressurized with gaseous nitrogen supplied from the high pressure nitrogen cylinder. An emulsion droplet is placed on the test surface by using a fuel supply system. The tip of needle of the fuel supply system is fixed $10 \mathrm{~mm}$ above the test surface and its plunger is slowly depressed until the droplet detaches from the tip of needle by its own weight. The behavior of the emulsion droplet evaporating on the hot surface is recorded by using a video camera during its whole life. The image of the emulsion droplet on the video monitor is available for determining the waiting time for the onset of micro-explosion and elucidating how the emulsion droplet behaves and what happens inside the droplet. The number of image and data acquisition ranges from 30 to 50 times for each test run. The emulsion temperature is probed by using a Pt-PtRh (13\%) thermocouple of a diameter of $50 \mu \mathrm{m}$. The hot junction of thermocouple is located in the predetermined position above the test surface and its output signal is recorded by the data recorder.

Measurements are done under the conditions of the ambient pressure ranging from atmospheric pressure to $0.3 \mathrm{MPa}$, the surface temperature from $650 \mathrm{~K}$ to $800 \mathrm{~K}$ and the initial water content from 0.1 to 0.2 . The initial diameter of emulsion droplet is kept constant at $2 \mathrm{~mm}$ for all test conditions.

The Weibull analysis ${ }^{(10)}$ is applied to derive the distribution function of the waiting time for the onset of microexplosion.

$$
\begin{aligned}
& F=1-\exp \left[-\left(\frac{\tau}{\alpha}\right)^{m}\right] \\
& \tau_{t}=\tau+\tau_{0} \\
& \tau^{*}=\frac{\alpha}{m} \Gamma\left(\frac{1}{m}\right) \\
& \tau_{t}^{*}=\tau^{*}+\tau_{0} \\
& J=\frac{m \tau^{m-1}}{\alpha^{m}}
\end{aligned}
$$

where $F, \tau, m, \alpha, \tau_{t}, \tau_{0}, \tau^{*}, \tau_{t}^{*}$ and $J$ are the distribution function of waiting time, the waiting time, the shape parameter, the scale parameter, the total waiting time, the minimum waiting time, the mean waiting time, the mean total waiting time and the rate of micro-explosion, respectively. 


\section{Experimental Results and Discussion}

It has been reported that the phase separation, the separation of the base fuel and water, occurs in an emulsion droplet evaporating and burning on a hot surface at atmospheric pressure ${ }^{(8),(9)}$. It has not been revealed, however, whether the phase separation occurs or not, or how the separated phases behave at high ambient pressure. A photographic observation has been made of the behavior of liquid phase in an emulsion droplet. Figure 2 shows an image of $n$-hexadecane-based emulsion droplet at the ambient pressure of $0.3 \mathrm{MPa}$, the initial water content (hereinafter called as the water content) of 0.2 and the surface temperature of $800 \mathrm{~K}$. An opaque droplet is formed in the central core of the emulsion droplet, being enveloped by a transparent shell. The internal opaque droplet consists of coagulated water and surfactant, whereas the transparent shell corresponds to the base fuel. In all conditions of the present measurements, the phase separation is observed to occur prior to micro-explosion.

Figure 3 shows the effect of the ambient pressure on the distribution function of the total waiting time for the onset of micro-explosion of $n$-hexadecane-based emulsion

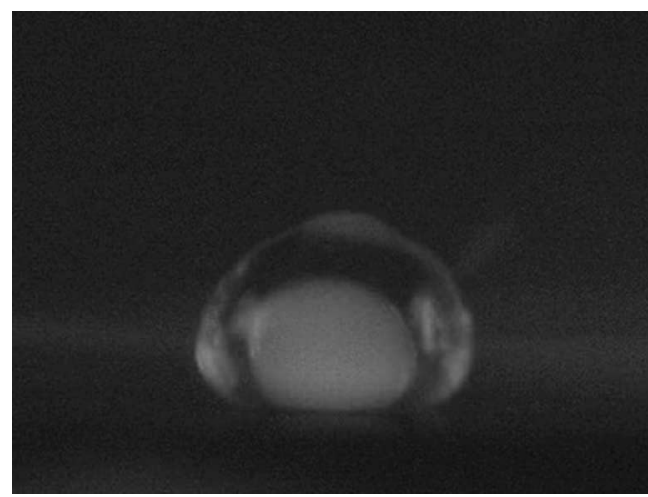

Fig. 2 Phase separation ( $n$-hexadecane/water, $C_{W}=0.2$, $\left.P_{a}=0.3 \mathrm{MPa}, T_{p}=800 \mathrm{~K}\right)$

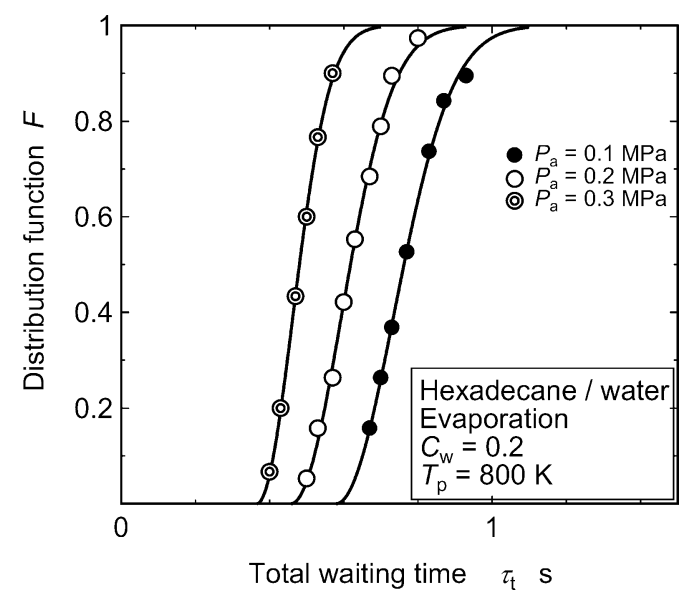

Fig. 3 Effect of ambient pressure on distribution function of total waiting time droplet at the water content of 0.2 and the surface temperature of $800 \mathrm{~K}$. The total waiting time in the abscissa is defined as the time period between the instant when the droplet is placed on the hot surface and the instant when the micro-explosion occurs. The slope of the distribution curve is rather moderate in the early and last stages and steep in the intermediate stage of the total waiting time. The increase in the ambient pressure causes the shift of the distribution curve toward the left-hand side, resulting in the reduced total waiting time $\tau_{t}$. The minimum waiting time $\tau_{0}$ in Eqs. (2) and (4) is defined as the time period between the time for the droplet to be placed on a hot surface and the time where the distribution curve crosses the abscissa in Fig. 3.

Figure 4 shows the Weibull plot of distribution function of the waiting time for the onset of micro-explosion. The abscissa represents the waiting time $\tau$. It is evident that the waiting time $\tau$ is correlated well with each straight line. The slope of the straight line appears to remain almost unchanged at different ambient pressures. The shape parameter of the Weibull distribution which corresponds to the slope of the straight line is approximately 2.0. This means that the onset of micro-explosion is classified into the wear-out type of the Weibull distribution in which the factors to cause the micro-explosion increase with the lapse of time.

Figure 5 shows the effect of the ambient pressure on the mean waiting time $\tau^{*}$ and the mean total waiting time $\tau_{t}^{*}$. The mean waiting time $\tau^{*}$ and the mean total waiting time $\tau_{t}^{*}$ are calculated by substituting the minimum waiting time $\tau_{0}$ determined in Fig. 3, and the shape parameter $m$ and the scale parameter $\alpha$ obtained in Fig. 4 into Eqs. (3) and (4). The mean waiting time $\tau^{*}$ and the mean total waiting time $\tau_{t}^{*}$ decrease with an increase in the ambient pressure.

Figure 6 shows the time histories of the rate of microexplosion. There is a linear relationship between the rate

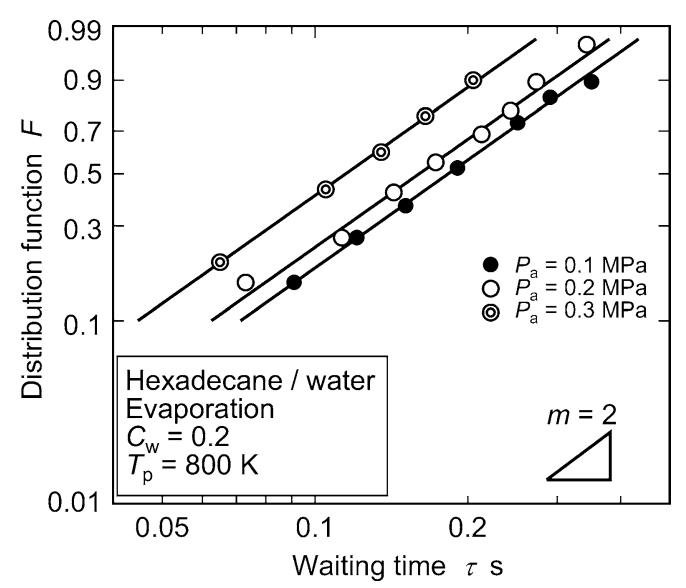

Fig. 4 Effect of ambient pressure on Weibull plot of waiting time 


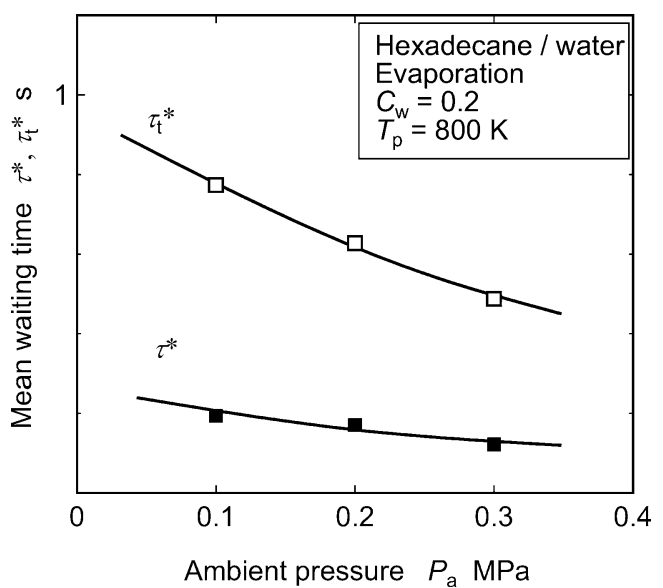

Fig. 5 Relationship between ambient pressure and mean waiting time

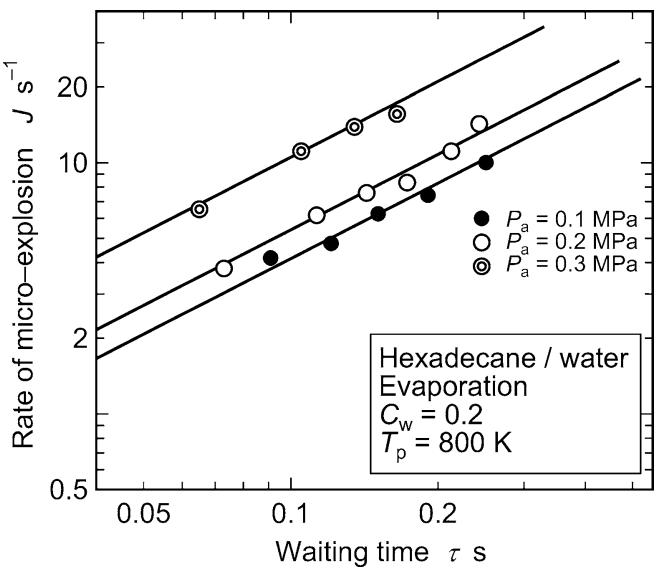

Fig. 6 Time histories of rate of micro-explosion

of micro-explosion $J$ and the waiting time $\tau$ at each ambient pressure on a logarithmic coordinate. The rate of micro-explosion increases with the lapse of time and its temporal rate of increase is high at high ambient pressure. This would be caused by the increased emulsion temperature at high ambient pressure.

Figure 7 shows the Weibull plot of distribution function of the waiting time for the onset of micro-explosion of emulsion droplets of three different base fuels at the water content of 0.2 , the ambient pressure of $0.2 \mathrm{MPa}$ and the surface temperature of $800 \mathrm{~K}$. The shape parameters which correspond to the slopes of these straight lines are approximately 2.0 and the onset of micro-explosion is classified into the wear-out type of the Weibull distribution. An increase in the saturation temperature of base fuel causes the shift of the distribution curve toward the lefthand side, resulting in the reduced waiting time. Figure 8 shows the effect of the ambient pressure on the mean waiting time $\tau^{*}$ and the mean total waiting time $\tau_{t}^{*}$ at the water content of 0.2 and the surface temperature of $800 \mathrm{~K}$ for $n$ hexadecane- and $n$-tetradecane-based emulsion droplets. The abscissa represents the reduced pressure $P_{a} / P_{c}$, the

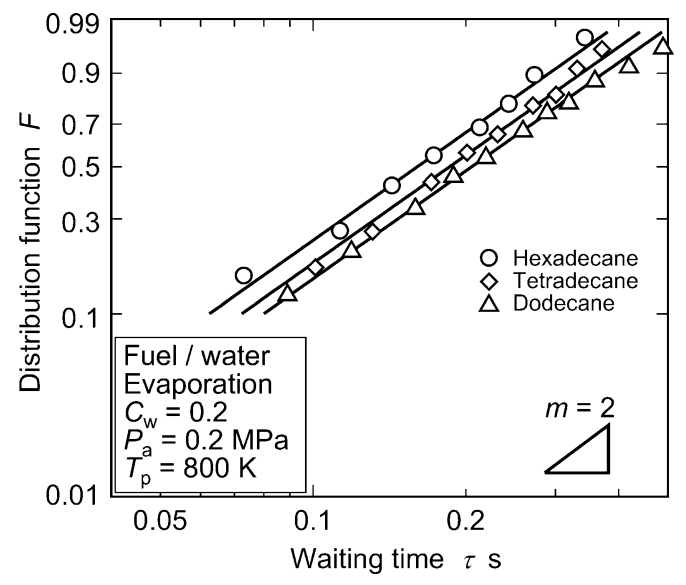

Fig. 7 Effect of base fuel on Weibull plot of waiting time

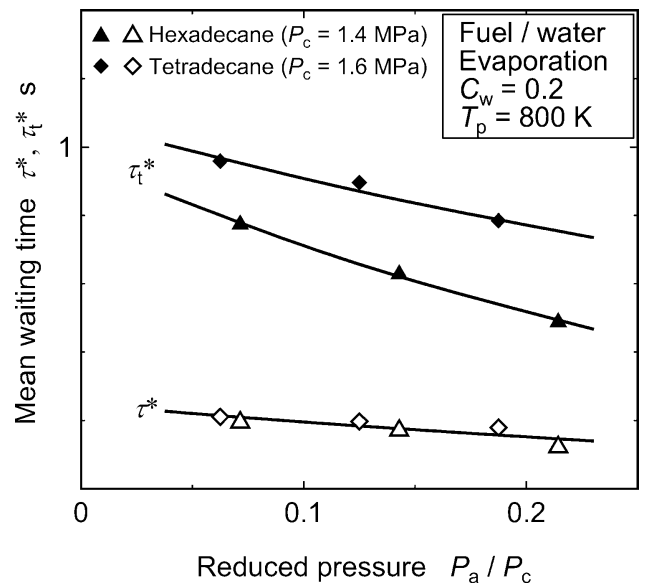

Fig. 8 Relationship between reduced pressure and mean waiting time

ambient pressure $P_{a}$ normalized by the critical pressure of base fuel $P_{c}$. Both the mean waiting time $\tau^{*}$ and the mean total waiting time $\tau_{t}^{*}$ decrease with an increase in the ambient pressure. There is no appreciable difference between the mean waiting times $\tau^{*}$ for two fuels. The mean total waiting time of $n$-hexadecane-based emulsion droplet is shorter than that of $n$-tetradecane-based emulsion droplet. Figure 9 shows the relationship between the saturation temperature and the mean waiting time $\tau^{*}$ and the mean total waiting time $\tau_{t}^{*}$. The mean waiting time $\tau^{*}$ or the mean total waiting time $\tau_{t}^{*}$ is correlated with a single curve for different base fuels and the ambient pressures. The increase in the saturation temperature causes the reduced mean waiting time $\tau^{*}$ and mean total waiting time $\tau_{t}^{*}$.

Figure 10 shows the effect of the water content on the Weibull distribution of the waiting time at the ambient pressure of $0.3 \mathrm{MPa}$ and the surface temperature of $800 \mathrm{~K}$. The slopes of two straight lines which correspond to different water content are almost equal. The shape parameter is approximately 2.0. The onset of micro-explosion is classified into the wear-out type of the Weibull distribution. The distribution curve shifts to the left-hand side 


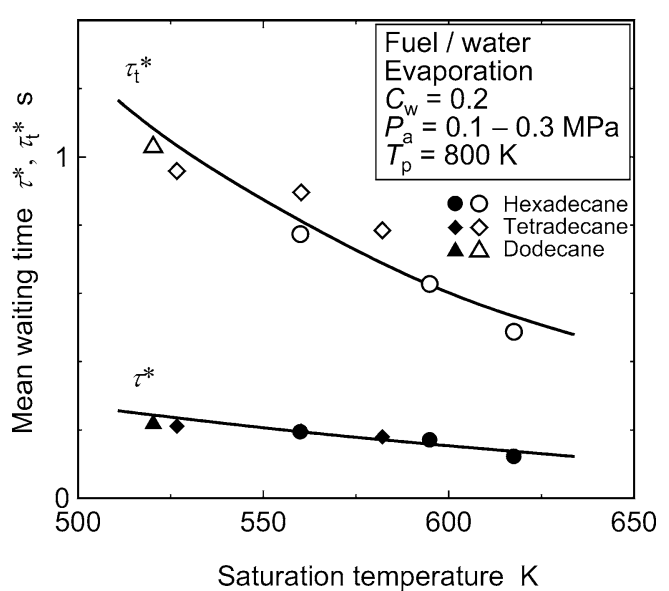

Fig. 9 Relationship between saturation temperature and mean waiting time

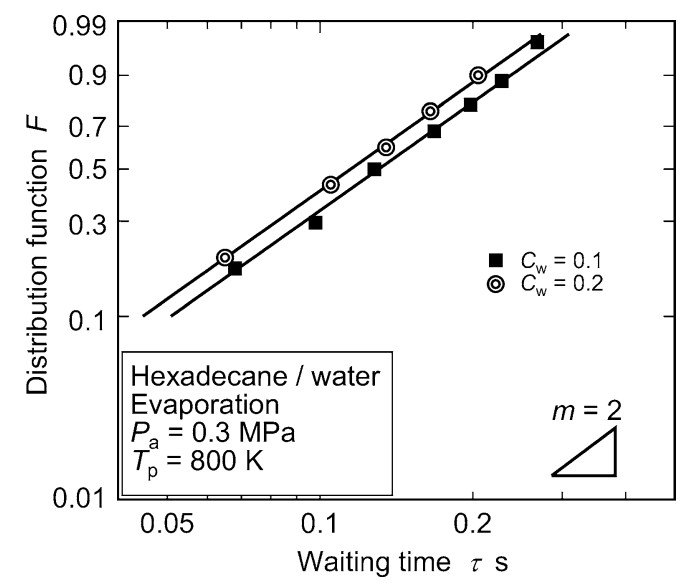

Fig. 10 Effect of water content on Weibull plot of waiting time

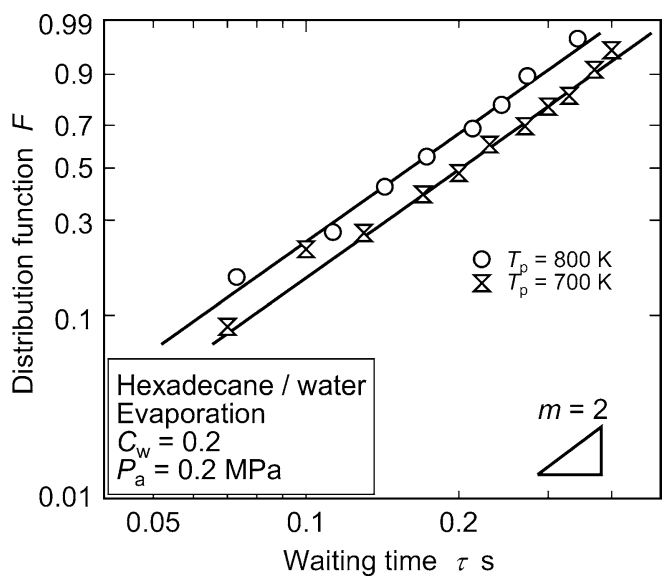

Fig. 11 Effect of surface temperature on Weibull plot of waiting time

with an increase in the water content, resulting in the reduced waiting time. Figure 11 shows the effect of the surface temperature on the Weibull distribution of the waiting time at the ambient pressure of $0.2 \mathrm{MPa}$ and the water content of 0.2 . The base fuel is $n$-hexadecane. The slopes of two straight lines at two different surface temperatures

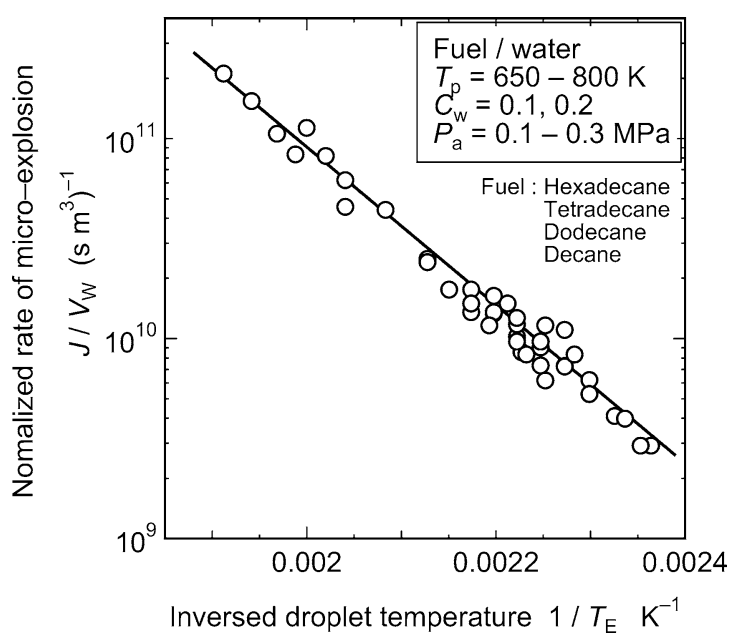

Fig. 12 Relationship between the rate of micro-explosion and the emulsion temperature

are approximately 2.0. The distribution curve shifts to the left-hand side with an increase in the surface temperature.

The rate of nucleation is expressed as a function of temperature in the homogeneous nucleation theory and the heterogeneous nucleation theory ${ }^{(3)}$. It is also reported that the rate of micro-explosion of an emulsion droplet burning in a quiescent air under microgravity and normal gravity conditions is correlated well with the emulsion temperature $^{(3)}$. An attempt is made to express the rate of microexplosion as a function of the emulsion temperature at the onset of micro-explosion. Figure 12 shows the relationship between the rate of micro-explosion $J$ normalized by the water volume $V_{W}$ and the emulsion temperature $T_{E}$. The emulsion temperature $T_{E}$ and the water volume $V_{W}$ are measured immediately prior to the onset of microexplosion. The surface temperature ranges from $650 \mathrm{~K}$ to $800 \mathrm{~K}$, the water content from 0.1 to 0.2 and the ambient pressure from $0.1 \mathrm{MPa}$ to $0.3 \mathrm{MPa}$. The results of emulsion droplets evaporating and burning at atmospheric pressure are referred to Ref. (9). It is evident that all the data are along a single straight line. From these results, the following empirical equation is derived for the rate of micro-explosion within the range of conditions of the present experiments.

$$
J=K V_{W} \exp \left[-\frac{A}{T_{E}}\right]
$$

where $K$ and $A$ are empirical coefficients. The waiting time depends on base fuel, the water content, the surface temperature and the ambient pressure. It is indicated, however, that the rate of micro-explosion $J$ normalized by the water volume is correlated well with the emulsion temperature $T_{E}$ at the onset of micro-explosion. Equation (6) has the same form as the equation of the rate of microexplosion for an emulsion droplet burning in a quiescent $\operatorname{air}^{(3)}$. From these results, it is implied that the mechanism of the onset of micro-explosion for an emulsion droplet 
evaporating and burning on a hot surface would be common to that for an emulsion droplet burning in a quiescent air.

\section{Conclusions}

An experimental study has been made of the microexplosion during Leidenfrost evaporation of an emulsion droplet on a hot surface in high pressure gaseous environments. Photographic observations are made to study how the emulsion droplet behaves and what happens inside the droplet and to measure the waiting time for the onset of micro-explosion. The Weibull analysis is applied to obtain the distribution function of the waiting time for the onset of micro-explosion and to derive the formula for the rate of micro-explosion as a function of the water volume and emulsion temperature.

The primary conclusions reached in the present study are as follows.

(1) The phase separation occurs in the emulsion droplet in high pressure gaseous environments as well as atmospheric pressure.

(2) The waiting time for the onset of microexplosion is correlated with the wear-out type of the Weibull distribution of the shape parameter two.

(3) The waiting time for the onset of microexplosion decreases with an increase in the ambient pressure.

(4) Both the mean waiting time and the mean total waiting time are correlated as a function of the saturation temperature of base fuel with individual single curves at various ambient pressures and base fuels.

(5) The waiting time for the onset of microexplosion decreases with an increase in the saturation temperature of base fuel, the water content and the surface temperature.

(6) The empirical equation is derived for the rate of micro-explosion as a function of the emulsion temperature and the water volume.

$$
J=K V_{W} \exp \left[-\frac{A}{T_{E}}\right]
$$

\section{References}

( 1 ) Dryer, F.L., Water Addition to Practical Combustion Systems - Concepts and Applications, Proceedings of the Combustion Institute, Vol.16 (1977), pp.279-295.

(2) Law, C.K., Recent Advances in Droplet Vaporization and Combustion, Progress in Energy and Combustion Science, Vol.8 (1982), pp.171-202.

( 3 ) Kadota, T. and Yamasaki, H., Recent Advances in the Combustion of Water Fuel Emulsion, Progress in Energy and Combustion Science, Vol.28 (2002), pp.385404.

( 4 ) Kimoto, K., Owashi, Y. and Omae, Y., The Vaporizing Behavior of the Oil Droplet of Water-in-Oil Emulsions on the Hot Surface, Trans. Jpn. Soc. Mech. Eng., (in Japanese), Vol.52, No.477, B (1986), pp.2275-2284.

( 5 ) Yoshimoto, Y., Tsukahara, M. and Murayama, T., Studies on the Microexplosion of Emulsified Fuels (1st Report, Effects of Fuel Properties, Water Contents, and Particle Sizes on the Microexplosion on a Hot Surface, Trans. Jpn. Soc. Mech. Eng., (in Japanese), Vol.55, No.519, B (1989), pp.3538-3543.

(6) Avedisian, C.T. and Fatehi, M., An Experimental Study of the Leidenfrost Evaporation Characteristics of Emulsified Liquid Droplets, International Journal of Heat and Mass Transfer, Vol.31, No.8 (1988), pp.15871603.

( 7 ) Chung, S.H. and Kim, J.C., An Experiment on Vaporization and Microexplosion of Emulsion Fuel Droplets on a Hot Surface, Proceedings of the Combustion Institute, Vol.23 (1990), pp.1431-1435.

( 8 ) Tanaka, H., Teraji, S., Yamasaki, H., Segawa, D. and Kadota, T., Statistical Analysis of Micro-Explosion of an Emulsion Droplet Evaporating and Burning on a Hot Surface, Trans. Jpn. Soc. Mech. Eng., (in Japanese), Vol.70, No.694, B (2004), pp.1577-1582.

( 9 ) Tanaka, H., Yamasaki, H., Teraji, S., Segawa, D. and Kadota, T., Effects of Fuel Properties, Water Contents and Surface Temperatures on Micro-Explosion of Emulsion Droplets Burning on a Hot Surface, Trans. Jpn. Soc. Mech. Eng., (in Japanese), Vol.71, No.702, B (2005), pp.690-695.

(10) Weibull, W., A Statistical Distribution Function of Wide Applicability, Journal of Applied Mechanics, Vol.18 (1951), pp.293-297. 